Bull. Korean Math. Soc. 51 (2014), No. 1, pp. 129-137

http://dx.doi.org/10.4134/BKMS.2014.51.1.129

\title{
ORIGIN-SYMMETRIC CONVEX BODIES WITH MINIMAL MAHLER VOLUME IN $\mathbb{R}^{2}$
}

\author{
Youjiang Lin And Gangsong Leng
}

\begin{abstract}
In this paper, a new proof of the following result is given: The product of the volumes of an origin-symmetric convex bodies $K$ in $\mathbb{R}^{2}$ and of its polar body is minimal if and only if $K$ is a parallelogram.
\end{abstract}

\section{Introduction}

A well-known problem in the theory of convex sets is to find a lower bound for the product of volumes $\mathcal{P}(K)=V(K) V\left(K^{*}\right)$, which is called the Mahler volume of $K$, where $K$ is an $n$-dimensional origin symmetric convex body and $K^{*}$ is the polar body of $K$ (see definition in Section 2). Is it true that we always have

$$
\mathcal{P}(K) \geq \mathcal{P}\left(B_{\infty}^{n}\right),
$$

where $B_{\infty}^{n}=\left\{x \in \mathbb{R}^{n}:\left|x_{i}\right| \leq 1,1 \leq i \leq n\right\}$ ? It was conjectured by $\mathrm{K}$. Mahler in [8] and K. Mahler himself in [9] proved the conjecture for $n=2$ and S. Reisner in [12] showed that parallelograms are the only minimizers. The $n=3$ case is still open. J. Saint-Raymond in [15], for $n>3$, exhibited convex bodies, different than $B_{\infty}^{n}$ and its polar body, for which (1.1) is an equality.

For some particular classes of convex symmetric bodies in $\mathbb{R}^{n}$, a sharper estimate for the lower bound of $\mathcal{P}(K)$ has been obtained. If $K$ is an affine image of convex body symmetric with respect to a coordinate hyperplane, J. Saint-Raymond in [15] proved that $\mathcal{P}(K) \geq 4^{n} / n$ !; the equality case, obtained for $1-\infty$ spaces, is discussed in [10] and [13]. When $K$ is a zonoid (limits of finite Minkowski sums of line segments), it was proved by S. Reisner in [11], [12] and [5] that the same inequality holds, with equality if and only if $K$ is an $n$-cube.

Received November 22, 2012; Revised July 16, 2013.

2010 Mathematics Subject Classification. 52A10, 52A40.

Key words and phrases. convex body, polar body, Mahler conjecture, polytopes.

The authors would like to acknowledge the support from China Postdoctoral Science Foundation Grant 2013M540806, National Natural Science Foundation of China under grant 11271244 and National Natural Science Foundation of China under grant 11271282 and the 973 Program 2013CB834201. 
Using the theory of cotype (which roughly speaking controls the size of long random sums in a normed vector space), J. Bourgain and V. D. Milman in [1] proved that there exists a universal constant $c>0$ such that $\mathcal{P}(K) \geq c^{n} \mathcal{P}\left(B_{\infty}^{n}\right)$, which is now known as the reverse Santaló inequality. A slightly weaker "lowtech" bound of $\mathcal{P}(K) \geq\left(\log _{2} n\right)^{-n} \mathcal{P}\left(B_{2}^{n}\right), n \geq 4$, was given by G. Kuperberg in [6], using only elementary methods. The best result currently known is again by G. Kuperberg in [7], who showed that the lower bound is roughly $(\pi / 4)^{n}$ times the product-volume of a cube by using some Gauss-type linking integrals associated to a Minkowski metric in $\mathbb{R}^{2 n}$.

The Mahler conjecture is still open even in the three-dimensional case. Terence Tao in [17] made an excellent remark about the open question.

In the paper, we give a new proof of Mahler conjecture in $\mathbb{R}^{2}$.

Theorem 1.1. If $K \subset \mathbb{R}^{2}$ is an origin-symmetric convex body, then

$$
\mathcal{P}(K) \geq 8,
$$

with equality if and only if $K$ is a square or diamond.

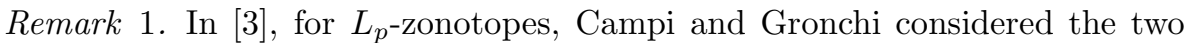
quantities $\frac{V\left(Z_{1}\right)}{V\left(Z_{p}\right)}$ and $V\left(Z_{1}\right) V\left(Z_{p}^{*}\right)$ and showed that both are minimized when $Z_{1}$ is a parallelotope, the second fact gave an interesting new proof of Mahler's conjecture in $\mathbb{R}^{2}$. The technique used by Campi and Gronchi is based on a method introduced by Rogers and Shephard in [14] and previous results proved by Campi and Gronchi in [2, 4] (They used the shadow system introduced by Rogers and Shephard). Our proof is different from the previous proof done by Campi and Gronchi, we first reduce to the problem to the case when $K$ has an axis of symmetry $D$ and uses as a parameter the coordinate on $D$ of the point of $K$ with maximal distance to $D$.

\section{Definitions, notation, and preliminaries}

As usual, $S^{n-1}$ denotes the unit sphere, $B^{n}$ the unit ball centered at the origin, $o$ the origin and $\|\cdot\|$ the norm in Euclidean $n$-space $\mathbb{R}^{n}$. If $x, y \in \mathbb{R}^{n}$, then $\langle x, y\rangle$ is the inner product of $x$ and $y$.

If $K$ is a set, $\partial K$ is its boundary, int $K$ is its interior, and conv $K$ denotes its convex hull. Let $\mathbb{R}^{n} \backslash K$ denote the complement of $K$, i.e., $\mathbb{R}^{n} \backslash K=\{x \in$ $\left.\mathbb{R}^{n}: x \notin K\right\}$. If $K$ is a $n$-dimensional convex subset of $\mathbb{R}^{n}$, then $V(k)$ is its volume $V_{n}(K)$.

Let $\mathcal{K}^{n}$ denote the set of convex bodies (compact, convex subsets with nonempty interiors) in $\mathbb{R}^{n}$. Let $\mathcal{K}_{o}^{n}$ denote the subset of $\mathcal{K}^{n}$ that contains the origin in its interior. Let $h(K, \cdot): S^{n-1} \rightarrow \mathbb{R}$, denote the support function of $K \in \mathcal{K}_{o}^{n}$; i.e.,

$$
h(K, u)=\max \{u \cdot x: x \in K\}, u \in S^{n-1},
$$

and let $\rho(K, \cdot): S^{n-1} \rightarrow \mathbb{R}$, denote the radial function of $K \in \mathcal{K}_{o}^{n}$; i.e.,

$$
\rho(K, u)=\max \{\lambda \geq 0: \lambda u \in K\}, u \in S^{n-1} .
$$


If $K \in K_{o}^{n}$, we define the polar body $K^{*}$ of $K$ by

$$
K^{*}=\left\{x \in \mathbb{R}^{n}: x \cdot y \leq 1, \forall y \in K\right\} .
$$

It is easy to verify that (see p. 44 in [16])

$$
h\left(K^{*}, u\right)=\frac{1}{\rho(K, u)} \text { and } \rho\left(\mathrm{K}^{*}, \mathrm{u}\right)=\frac{1}{\mathrm{~h}(\mathrm{~K}, \mathrm{u})} .
$$

\section{Main result and its proof}

Lemma 3.1. For any origin symmetric convex body $K \subset \mathbb{R}^{n}, \mathcal{P}(K)$ is linear invariant, that is, for every linear transformation $A: \mathbb{R}^{n} \rightarrow \mathbb{R}^{n}$, we have $\mathcal{P}(A K)=\mathcal{P}(K)$.

Proof. For any $u \in S^{n-1}$, we have

$$
\rho\left((A K)^{*}, u\right)=\frac{1}{h(A K, u)}=\frac{1}{h\left(K, A^{t} u\right)}=\rho\left(K^{*}, A^{t} u\right)=\rho\left(A^{-t} K^{*}, u\right) .
$$

Hence, $(A K)^{*}=A^{-t} K^{*}$, therefore

$$
\begin{aligned}
& \mathcal{P}(A K)=V(A K) V\left((A K)^{*}\right)=V(A K) V\left(A^{-t} K^{*}\right) \\
& =|A|\left|A^{-t}\right| V(K) V\left(K^{*}\right)=V(K) V\left(K^{*}\right)=\mathcal{P}(K) .
\end{aligned}
$$

Lemma 3.2. In $\mathbb{R}^{2}$, for any origin symmetric convex body $K_{0}$, there exists a convex body $K$, symmetric with respect to $X$-axis $O X$ and $K \cap O X=[-a, a]$, satisfying $\mathcal{P}(K)=\mathcal{P}\left(K_{0}\right)$.

Proof. Since $K_{0}$ is origin symmetric, any line through origin $O$ cuts the boundary of $K_{0}$ into two points with parallel support lines to $K_{0}$. Since $\mathcal{P}\left(K_{0}\right)$ is affinely invariant, we can suppose that these parallel support lines are orthogonal to $\mathrm{X}$-axis. We define now

$$
K=\left\{(x, y) \mid(x,|y|) \in K_{0}\right\} .
$$

It is clear that $K$ is symmetric with respect to $\mathrm{X}$-axis and $V(K)=V\left(K_{0}\right)$, thus we just need to prove $V\left(K^{*}\right)=V\left(K_{0}{ }^{*}\right)$.

$$
\begin{aligned}
V\left(K_{0}^{*}\right) & =\frac{1}{2} \int_{S^{1}} \rho\left(K_{0}^{*}, u\right)^{2} d u \\
& =\frac{1}{2} \int_{S^{1}} h\left(K_{0}, u\right)^{-2} d u \\
& =\int_{S_{+}^{1}} h\left(K_{0}, u\right)^{-2} d u \\
& =\int_{S_{+}^{1}} h(K, u)^{-2} d u \\
& =\frac{1}{2} \int_{S^{1}} h(K, u)^{-2} d u
\end{aligned}
$$




$$
\begin{aligned}
& =\frac{1}{2} \int_{S^{1}} \rho(K *, u)^{2} d u \\
& =V\left(K^{*}\right),
\end{aligned}
$$

where $S_{+}^{1}=S^{1} \cap\{(x, y) \mid y \geq 0\}$, which completes the proof.

We define function $f(x)=\max \{y \mid(x, y) \in K\}$, then $f(x)$ is a concave non-negative function on $[-a, a]$ and $K=\{(x, y)|x \in[-a, a]| y \mid, \leq f(x)\}$. We have the following theorem.

Lemma 3.3. If $K=\{(x, y)|x \in[-a, a]| y \mid, \leq f(x)\}$, where $f(x)$ is a concave non-negative function on $[-a, a]$, then

$$
K^{*}=\left\{\left(x^{\prime}, y^{\prime}\right)\left|x^{\prime} \in\left[-\frac{1}{a}, \frac{1}{a}\right],\right| y^{\prime} \mid \leq f^{*}\left(x^{\prime}\right)\right\},
$$

where

$$
f^{*}\left(x^{\prime}\right)=\inf \left\{\frac{1-x x^{\prime}}{f(x)} \mid x \in[-a, a]\right\} .
$$

Proof. We firstly prove that $f^{*}$ is also a concave non-negative function on $\left[-\frac{1}{a}, \frac{1}{a}\right]$. Because that $f(x) \geq 0$ and $1-x x^{\prime} \geq 0$, thus $f^{*}\left(x^{\prime}\right) \geq 0$ for any $x^{\prime} \in\left[-\frac{1}{a}, \frac{1}{a}\right]$. By the definition of $f^{*}\left(x^{\prime}\right)$, for any $-\frac{1}{a} \leq x_{1}^{\prime}, x_{2}^{\prime} \leq \frac{1}{a}$ and $0<\lambda<1$, we have

$$
\begin{aligned}
& f^{*}\left((1-\lambda) x_{1}^{\prime}+\lambda x_{2}^{\prime}\right) \\
= & \inf \left\{\frac{1-x\left((1-\lambda) x_{1}^{\prime}+\lambda x_{2}^{\prime}\right)}{f(x)} \mid x \in[-a, a]\right\} \\
= & \inf \left\{\frac{\left.(1-\lambda)\left(1-x x_{1}^{\prime}\right)+\lambda\left(1-x x_{2}^{\prime}\right)\right)}{f(x)} \mid x \in[-a, a]\right\} \\
\geq & (1-\lambda) \inf \left\{\frac{1-x x_{1}^{\prime}}{f(x)} \mid x \in[-a, a]\right\}+\lambda \inf \left\{\frac{1-x x_{2}^{\prime}}{f(x)} \mid x \in[-a, a]\right\} \\
(3.2)= & (1-\lambda) f^{*}\left(x_{1}^{\prime}\right)+\lambda f^{*}\left(x_{2}^{\prime}\right),
\end{aligned}
$$

which implies that $f^{*}$ is a concave function.

Next, we let

$$
K_{1}=\left\{\left(x^{\prime}, y^{\prime}\right)\left|x^{\prime} \in\left[-\frac{1}{a}, \frac{1}{a}\right],\right| y^{\prime} \mid \leq f^{*}\left(x^{\prime}\right)\right\} .
$$

On the one hand, we prove $K_{1} \subset K^{*}$, for any $\left(x^{\prime}, y^{\prime}\right) \in K_{1}$ and $(x, y) \in K$, we have

$$
\begin{aligned}
& x x^{\prime}+y y^{\prime} \\
\leq & x x^{\prime}+y f^{*}\left(x^{\prime}\right) \\
\leq & x x^{\prime}+y \frac{1-x x^{\prime}}{f(x)} \\
\leq & x x^{\prime}+f(x) \frac{1-x x^{\prime}}{f(x)}
\end{aligned}
$$




$$
\leq 1
$$

which implies that $K_{1} \subset K^{*}$.

On the other hand, we prove $K^{*} \subset K_{1}$. Let $\left(x^{\prime}, y^{\prime}\right) \in K^{*}$ and $-a<x<a$, then $(x, 0) \in K$, we have $x x^{\prime} \leq 1$ for any $-a<x<a$, hence $-\frac{1}{a} \leq x^{\prime} \leq \frac{1}{a}$. For any $(x, f(x)) \in K$, we have $x x^{\prime}+f(x) y^{\prime} \leq 1$, then $\left|f(x) y^{\prime}\right| \leq 1-x x^{\prime}$, thus $\left|y^{\prime}\right| \leq \frac{1-x x^{\prime}}{f(x)}$, thus $\left|y^{\prime}\right| \leq \inf \left\{\frac{1-x x^{\prime}}{f(x)} \mid x \in[a, b]\right\}=f^{*}\left(x^{\prime}\right)$, which implies that $K^{*} \subset K_{1}$.

From the above lemma, we have

$$
\mathcal{P}(K)=4\left(\int_{-a}^{a} f(x) d x\right) \cdot\left(\int_{-\frac{1}{a}}^{\frac{1}{a}} f^{*}(x) d x\right) .
$$

Let now $t_{0} \in[-a, a]$, such that $f\left(t_{0}\right)=M$ is maximal on $[-a, a]$; then for any $x^{\prime} \in\left[0, \frac{1}{a}\right]$, since

$$
\frac{1-x^{\prime} x}{f(x)} \geq \frac{1-x^{\prime} t_{0}}{f\left(t_{0}\right)}
$$

for any $x \in\left[-a, t_{0}\right]$, thus

$$
f^{*}\left(x^{\prime}\right)=\inf \left\{\frac{1-x^{\prime} x}{f(x)} \mid x \in\left[t_{0}, a\right]\right\}
$$

similarly for $x^{\prime} \in\left[-\frac{1}{a}, 0\right]$,

$$
f^{*}\left(x^{\prime}\right)=\inf \left\{\frac{1-x^{\prime} x}{f(x)} \mid x \in\left[-a, t_{0}\right]\right\} .
$$

It follows that if we set

$$
\begin{aligned}
& F_{1}=\left\{(x, y) \in K \quad \mid y \geq 0, x \geq t_{0}\right\} \\
& F_{2}=\left\{(x, y) \in K \quad \mid y \geq 0, x \leq t_{0}\right\} \\
& F_{1}^{*}=\left\{(x, y) \in K^{*} \mid y \geq 0, x \geq 0\right\} \\
& F_{2}^{*}=\left\{(x, y) \in K^{*} \mid y \geq 0, x \leq 0\right\}
\end{aligned}
$$

and let $u_{i}=\frac{2\left|F_{i}\right|}{M}, i=1,2$, we have

$$
\begin{gathered}
M u_{1} \geq\left(x-t_{0}\right) M+f(x)\left(a-t_{0}\right) \text { for } x \in\left[t_{0}, a\right] \\
M u_{2} \geq\left(t_{0}-x\right) M+f(x)\left(a+t_{0}\right) \text { for } x \in\left[-a, t_{0}\right] .
\end{gathered}
$$

Lemma 3.4. Let $v_{i}=2\left|F_{i}^{*}\right| M, i=1,2$. Then we can get the following three inequalities:

$$
\begin{gathered}
v_{1}\left(u_{1}+t_{0}\right) \geq 2-\frac{t_{0}}{a}, v_{2}\left(u_{2}-t_{0}\right) \geq 2+\frac{t_{0}}{a} \\
a-t_{0} \leq u_{1} \leq 2\left(a-t_{0}\right), a+t_{0} \leq u_{2} \leq 2\left(a+t_{0}\right)
\end{gathered}
$$




$$
\frac{1}{a} \leq v_{1} \leq \frac{1}{a}\left(2-\frac{t_{0}}{a}\right), \frac{1}{a} \leq v_{2} \leq \frac{1}{a}\left(2+\frac{t_{0}}{a}\right) .
$$

Proof. Let

and

$$
A_{1}=\left(x_{1}, y_{1}\right)=\left(\frac{1}{u_{1}+t_{0}}, \frac{a-t_{0}}{M\left(u_{1}+t_{0}\right)}\right)
$$

$$
A_{2}=\left(x_{2}, y_{2}\right)=\left(\frac{-1}{u_{2}-t_{0}}, \frac{t_{0}+a}{M\left(u_{2}-t_{0}\right)}\right),
$$

next, we prove $A_{i} \in F_{i}^{*}$ and $0 \leq y_{i} \leq f^{*}\left(x_{i}\right) i=1,2$. Because $2\left|F_{1}\right|>M\left(a-t_{0}\right)$ and $x_{1}=\frac{1}{u_{1}+t_{0}}=\frac{M}{2\left|F_{1}\right|+M t_{0}}$, hence $0<x_{1}<\frac{1}{a}$ and $y_{1}>0$. By $y_{1}=\frac{a-t_{0}}{M\left(u_{1}+t_{0}\right)}$ and $f^{*}\left(x_{1}\right)=\inf \left\{\frac{1-x x_{1}}{f(x)} \mid x \in\left[t_{0}, a\right]\right\}$, we prove that $\frac{a-t_{0}}{M\left(u_{1}+t_{0}\right)} \leq \frac{1-x x_{1}}{f(x)}$ for any $x \in\left[t_{0}, a\right]$.

$$
\begin{aligned}
& \frac{a-t_{0}}{M\left(u_{1}+t_{0}\right)} \leq \frac{1-x x_{1}}{f(x)} \\
\Longleftrightarrow & f(x)\left(a-t_{0}\right) \leq\left(1-x x_{1}\right) M\left(u_{1}+t_{0}\right) \\
\Longleftrightarrow & f(x)\left(a-t_{0}\right) \leq\left(1-x x_{1}\right) M\left(\frac{2\left|F_{1}\right|}{M}+t_{0}\right) \\
\Longleftrightarrow & f(x)\left(a-t_{0}\right) \leq\left(1-x x_{1}\right)\left(2\left|F_{1}\right|+M t_{0}\right) \\
\Longleftrightarrow & f(x)\left(a-t_{0}\right) \leq\left(1-\frac{x}{u_{1}+t_{0}}\right)\left(2\left|F_{1}\right|+M t_{0}\right) \\
\Longleftrightarrow & f(x)\left(a-t_{0}\right) \leq\left(1-\frac{x}{\frac{2\left|F_{1}\right|}{M}+t_{0}}\right)\left(2\left|F_{1}\right|+M t_{0}\right) \\
\Longleftrightarrow & f(x)\left(a-t_{0}\right) \leq\left(1-\frac{M x}{2\left|F_{1}\right|+M t_{0}}\right)\left(2\left|F_{1}\right|+M t_{0}\right) \\
\Longleftrightarrow & f(x)\left(a-t_{0}\right) \leq 2\left|F_{1}\right|+M t_{0}-M x \\
\Longleftrightarrow & 2\left|F_{1}\right| \geq M\left(x-t_{0}\right)+f(x)\left(a-t_{0}\right)
\end{aligned}
$$

which is (3.12), hence $y_{1} \leq f^{*}\left(x_{1}\right)$, hence $A_{1} \in F_{1}^{*}$. Similarly, we can get $A_{2} \in F_{2}^{*}$.

According to Figure 3 , we can get $2\left|F_{1}^{*}\right| \geq x_{1} f^{*}(0)+f^{*}\left(x_{1}\right) \frac{1}{a}$, and because $f^{*}(0)=\frac{1}{M}$ and $y_{1} \leq f^{*}\left(x_{1}\right)$, hence

$$
\frac{v_{1}}{M} \geq \frac{x_{1}}{M}+\frac{y_{1}}{a} .
$$

Similarly, we can get

$$
\frac{v_{2}}{M} \geq-\frac{x_{2}}{M}+\frac{y_{2}}{a} .
$$

The above two inequalities are equivalent to (3.14).

From Figure 2, we can get (3.15) easily.

Next, we prove (3.16). Because

$$
f^{*}(x)=\inf \left\{\frac{1-x t}{f(t)} \mid t \in[-a, a]\right\} \leq \frac{1-x t_{0}}{M}
$$




$$
\begin{aligned}
& \Longrightarrow \quad \int_{0}^{\frac{1}{a}} f^{*}(x) \leq \int_{0}^{\frac{1}{a}} \frac{1-x t_{0}}{M} d x \\
& \Longrightarrow \quad\left|F_{1}\right| \leq \frac{1}{2 M} \cdot \frac{1}{a}\left(2-\frac{t_{0}}{a}\right)
\end{aligned}
$$

and $\left|F_{1}\right| \geq \frac{1}{2} \frac{1}{M} \frac{1}{a}$, hence

$$
\frac{1}{a} \leq v_{1} \leq \frac{1}{a}\left(2-\frac{t_{0}}{a}\right)
$$

Similarly,

$$
\begin{aligned}
& \int_{-\frac{1}{a}}^{0} f^{*}(x) \leq \int_{-\frac{1}{a}}^{0} \frac{1-x t_{0}}{M} d x \\
\Longrightarrow & \left|F_{2}\right| \leq \frac{1}{2 M} \cdot \frac{1}{a}\left(2+\frac{t_{0}}{a}\right)
\end{aligned}
$$

and $\left|F_{2}\right| \geq \frac{1}{2} \frac{1}{M} \frac{1}{a}$, hence

$$
\frac{1}{a} \leq v_{1} \leq \frac{1}{a}\left(2+\frac{t_{0}}{a}\right)
$$

We have proved (3.16).

Theorem 3.5. If $K_{0} \subset \mathbb{R}^{2}$ is an origin-symmetric convex body, then

$$
\mathcal{P}(K) \geq 8
$$

with equality if and only if $K$ is a square or diamond.

Proof. Let $K \subset \mathbb{R}^{2}$ be a convex body symmetric with respect to X-axis OX and $K \cap O X=[-a, a]$ and $\mathcal{P}(K)=\mathcal{P}\left(K_{0}\right)$. By (3.4), (3.14) and let $q=\frac{v_{2}}{v_{1}}$, we have

$$
\begin{aligned}
\mathcal{P}(K) & =\left(u_{1}+u_{2}\right)\left(v_{1}+v_{2}\right) \\
& =u_{1} v_{1}+u_{1} v_{2}+u_{2} v_{1}+u_{2} v_{2} \\
& \geq\left(2-\frac{t_{0}}{a}-v_{1} t_{0}\right)+u_{1} v_{2}+u_{2} v_{1}+\left(2+\frac{t_{0}}{a}+v_{2} t_{0}\right) \\
& =4-v_{1} t_{0}+u_{1} v_{2}+u_{2} v_{1}+v_{2} t_{0} \\
& =4+\frac{v_{2}}{v_{1}}\left(u_{1} v_{1}+v_{1} t_{0}\right)+\frac{v_{1}}{v_{2}}\left(u_{2} v_{2}-v_{2} t_{0}\right) \\
& \geq 4+q\left(2-\frac{t_{0}}{a}\right)+\frac{1}{q}\left(2+\frac{t_{0}}{a}\right) \\
& =4+\frac{1}{a}\left(\frac{1}{q}-q\right) t_{0}+2\left(q+\frac{1}{q}\right) .
\end{aligned}
$$

If $v_{2}>v_{1}$, then $q>1$ and $\frac{1}{q}-q<0$, then

(3.23) $\mathcal{P}(K) \geq 4+\frac{1}{a}\left(\frac{1}{q}-q\right) a+2\left(q+\frac{1}{q}\right)=4+q+\frac{3}{q} \geq 4+2 \sqrt{3}$,

and it follows from (3.16) that

$$
1<q=\frac{v_{2}}{v_{1}} \leq 2+\frac{t_{0}}{a} \leq 3 .
$$


If $v_{2}<v_{1}$, then $q<1$ and $\frac{1}{q}-q>0$, then

$$
\mathcal{P}(K) \geq 4+\frac{1}{a}\left(\frac{1}{q}-q\right)(-a)+2\left(q+\frac{1}{q}\right)=4+3 q+\frac{1}{q} \geq 4+2 \sqrt{3},
$$

and it follows from (3.16) that

$$
1>q=\frac{v_{2}}{v_{1}} \geq \frac{1}{2-\frac{t_{0}}{a}} \geq \frac{1}{3} .
$$

If $v_{2}=v_{1}$, we have

$$
\mathcal{P}(K) \geq 4+2\left(q+\frac{1}{q}\right) \geq 8 .
$$

In the following, we prove the case of equality in (3.12). If $K$ is a square or a diamond, it is clear that the equality holds. On the other hand, if the equality holds, then equalities in (3.14) hold, which implies that $K$ is a square or a diamond.

\section{References}

[1] J. Bourgain and V. D. Milman, New volume ratio properties for convex symmetric bodies in $\mathbb{R}^{n}$, Invent. Math. 88 (1987), no. 2, 319-340.

[2] S. Campi and P. Gronchi, The L $L^{p}$-Busemann-Petty centroid inequality, Adv. Math. 167 (2002), 128-141.

[3] — Volume inequalities for $L_{p}$-zonotopes, Mathematika 53 (2006), no. 1, 71-80.

[4] - On volume product inequalities for convex sets, Proc. Amer. Math. Soc. 134 (2006), no. 8, 2393-2402.

[5] Y. Gordon, M. Meyer, and S. Reisner, Zonoids with minimal volume-product - a new proof, Proc. Amer. Math. Soc. 104 (1988), no. 1, 273-276.

[6] G. Kuperberg, A low-technology estimate in convex geometry, Internat. Math. Res. Notices 1992 (1992), no. 9, 181-183.

[7] _ From the Mahler conjecture to Gauss linking integrals, Geom. Funct. Anal. 18 (2008), no. 3, 870-892.

[8] K. Mahler, Ein Übertragungsprinzip für konvexe Körper, Časopis Pyest. Mat. Fys. 68 (1939), 93-102.

[9] _ Ein Minimalproblem für konvexe Polygone, Mathematica (Zutphen) B. 7 (1939), 118-127.

[10] M. Meyer, Une caractérisation volumique de certains espaces normés de dimension finie, Israel J. Math. 55 (1986), no. 3, 317?-326.

[11] S. Reisner, Random polytopes and the volume-product of symmetric convex bodies, Math. Scand. 57 (1985), no. 2, 386-392.

[12] _ Zonoids with minimal volume-product, Math. Z. 192 (1986), no. 3, 339-346.

[13] _ Minimal volume product in Banach spaces with a 1-unconditional basis, J. London Math. Soc. 36 (1987), no. 1, 126-136.

[14] C. A. Rogers and G. C. Shephard, Some extremal problems for convex bodies, Mathematika 5 (1958), 93-102.

[15] J. Saint Raymond, Sur le volume des corps convexes symétriques, Initiation Seminar on Analysis: G. Choquet-M. Rogalski-J. Saint-Raymond, 20th Year: 1980/1981, Exp. No. 11, 25 pp., Publ. Math. Univ. Pierre et Marie Curie, 46, Univ. Paris VI, Paris, 1981

[16] R. Schneider, Convex Bodies: The Brunn-Minkowski Theory, Encyclopedia Math. Appl., vol. 44, Cambridge University Press, Cambridge, 1993. 
[17] T. Tao, Structure and Randomness, Pages from year one of a mathematical blog. American Mathematical Society, Providence, RI, 2008.

YOUJIANG LIN

School of Mathematical Sciences

PEKING UNIVERSITY

Beijing, 100871, P. R. China

AND

Department of Mathematics

Department of Mathematics

Shanghai University

Shanghai, 200444, P. R. China

E-mail address: lxyoujiang@126.com

Gangsong Leng

Department of Mathematics

Shanghai University

Shanghai, 200444, P. R. China

E-mail address: gleng@staff.shu.edu.cn 